\title{
Comments on purification in continuum games
}

\author{
Erik J. Balder
}

Accepted: 5 April 2007 / Published online: 9 May 2007

(C) Springer-Verlag 2007

\begin{abstract}
It is pointed out that Corollary 1 in a recent paper by Khan et al. (Int J Game Theory 34:91-104, 2006), presented there as an extension of the DvoretzkyWald-Wolfowitz theorem, is a special case of Lyapunov's theorem for Young measures (Balder in Rend Instit Mat Univ Trieste 31 Suppl. 1:1-69) It is also pointed out that Theorems 1-4 in Khan et al. (Int J Game Theory 34:91-104, 2006) follow from a single strong purification per se result that is already contained, as an implementation of that Lyapunov theorem for Young measures, in the proof of Theorem 2.2.1 in Balder (J Econ Theory 102:437-470, 2002).
\end{abstract}

Keywords Equilibrium distribution - Games with a continuum of players · Games with incomplete information - Games with private information - Large games . Lyapunov's theorem · Nash equilibrium · Purification · Young measures

\section{Introduction}

A well-known method to establish the existence of pure Nash equilibria for games with a measure space of players goes by means of the following proof-scheme:

Step 1: establish the existence of a mixed equilibrium.

Step 2: purify the mixed equilibrium, established in Step 1, by some purification device.

So Step 2 of the scheme concerns the purification of mixed equilibrium profiles, as opposed to what we shall call purification per se, that is to say, the purification of general mixed profiles. Nevertheless, for the execution of Step 2 one usually employs

E. J. Balder $(\varangle)$

Mathematical Institute, University of Utrecht, Budapestlaan 6,

P.O. Box 80.010, 3508 TA Utrecht, The Netherlands

e-mail: balder@math.uu.nl 
a purification device that has much greater generality. In the important situation where the measure space of players is non-atomic, such devices come in different but closely related forms, such as Lyapunov's theorem, Aumann's identity or the purification theorem by Dvoretzky, Wald and Wolfowitz, which extends Lyapunov's theorem (these results are recalled in the appendix to this paper-see Theorems A.1, A.2 and A.4). For instance, the important paper by Schmeidler (1973) uses Aumann's identity as its purification device. In Balder (2000, Theorem 5.10) the present author introduced a purification device that he called Lyapunov's theorem for Young measures (it is recalled below in Theorem 2.1). This result stems from Balder (1984, Theorem 1'), a result that already contains both the purification theorem of Dvoretzky et al. (1950) and Aumann's identity [see also (Balder 1985)]. Employing this purification device, the above two-step proof-scheme was applied in Balder (2002) to obtain Theorem 2.2.1 therein (for the reader's convenience this result is also recalled in the appendix-see Theorem A.5). The generality of this pure equilibrium existence result and its companion mixed equilibrium existence result (this is Theorem 2.1.1 of Balder (2002), which corresponds to Step 1 in the above scheme) is known to be considerable. This is not only due to a very general choice of topologies, but also due to the fact that Balder (2002) uses a continuum game model that is in internal-external form. This formal concept stems from Balder (1995). Games in internal-external form would appear to be a quite natural extension of the concept of games in strategic form and a large variety of games with a measure space of players/types is known to be representable in that form (Balder 1991, 1995, 1996, 1999, 2002); see also Angeloni and Martins-da-Rocha (2005) for recent discoveries of such representations. Martins-da-Rocha and Topuzu (2006) have recently extended the scope of the internal-external form model even further: by introducing artificial payoffs of a generalized Shafer-Sonnenschein variety (Shafer and Sonnenschein 1975) they have shown that the existence results from Balder (2002) can be extended to continuum games with non-ordered preferences.

In a recent paper Khan et al. (2006) gave four purification results for games with finitely many actions and a non-atomic measure space of players (or players' types). While their Theorems 1-2 concern purification per se, their Theorems 3-4 concern the purification of mixed equilibrium profiles as in Step 2 of the above proof-scheme. In the last line of Khan et al. (2006) its authors express the hope that their results "may have further application to more general settings", among which they include Balder (2002) and its references. The purpose of this paper is to point out that precisely the contrary is true: when specialized to finite action spaces, certain results in Balder (2002) already imply those of Khan et al. (2006). More precisely, we show the following:

(i) The principal purification device of Khan et al. (2006), presented as an extension of the original Dvoretzky-Wald-Wolfowitz theorem (see Theorem A.2 in the appendix), is a special case of Lyapunov's theorem for Young measures, i.e., the purification device used in Balder (2002).

(ii) The implementation of Lyapunov's theorem for Young measures in a game context, given in Step 4 on p. 465 of Balder (2002), already constitutes a very general result on purification per se. It implies the results on strong purification per se in (Khan et al. 2006, Theorems 1-2) and also improves the purification results in (Khan et al. 2006, Theorems 3-4). 
In Balder (2002), a paper focusing on existence, the above-mentioned implementation of Lyapunov's theorem for Young measures was only concerned with the purification of mixed equilibrium profiles, namely in Step 4 on (Balder 2002, p. 465). However, this purification device is so general as to cause Step 4 on (Balder 2002, p. 465) to apply also to the purification of an arbitrary mixed profile, i.e., to purification per se. Actually, it turns out that not only this Step 4 in the proof of Theorem 2.2.1 can be applied to the models considered in Khan et al. (2006), but also that pure equilibrium existence result itself; cf. Remark 3.2. So in addition this yields pure equilibrium existence in all four models studied in Khan et al. (2006) [recall that existence is an issue not addressed at all in Khan et al. (2006)].

The setup of this paper is as follows. In Sect. 2 we recall Lyapunov's theorem for Young measures (Theorem 2.1) and show how (Khan et al. 2006, Corollary 1), the principal tool of Khan et al. (2006), is its direct consequence (the latter result is recalled in the appendix-see Theorem A.3). In Sect. 3 we state the implementation of Lyapunov's theorem for Young measures in the internal-external form game model, as found in (Balder 2002, p. 465, Step 4), as a separate and very general result on purification per se. This is Theorem 3.1, which directly leads to Corollary 3.1. Subsequently, in Sect. 4 we adopt the finite action space setting of Khan et al. (2006) and demonstrate that Theorems 1-4 in Khan et al. (2006) all follow from Corollary 3.1. We do this by showing that each of the four game-theoretical models in Khan et al. (2006) can be reformulated as a game in internal-external form.

Finally, we observe that all the purification devices discussed depend heavily on Lyapunov's classical theorem. An interesting development in recent years, following path-breaking work in Kingman and Robertson (1968), has been the development of suitable infinite-dimensional versions of Lyapunov's theorem and concomitant purification results, based on non-injectivity conditions; see (Balder 2004; Khan and Sun 1996; Loeb and Sun 2006; Podczeck 2003; Podczek 2006; Rustichini and Yannelis 1991; Tourky and Yannelis 2001). For the present paper it is interesting to notice that some of these cited papers reiterate well-known parts of Young measure theory, apparently without realizing the connection.

\section{Lyapunov's theorem for Young measures}

Let $(T, \mathcal{T}, \mu)$ be a finite and non-atomic measure space and let $S$ be a metric space that forms a Borel-measurable subset of a complete separable and metric space (i.e., $S$ is a metrizable Suslin space). Let $\mathcal{B}(S)$ be the usual Borel $\sigma$-algebra on $S$ and let $\operatorname{Prob}(S)$ be the set of all probability measures on $(S, \mathcal{B}(S))$. Recall that the support supp $v$ of a probability measure $v \in \operatorname{Prob}(S)$ is defined as the intersection of all closed sets in $S$ of $v$-measure 1 (equivalently, supp $v$ is the complement of the union of all open $v$-null sets).

Recall that a transition probability (also called Young measure) with respect to $(T, \mathcal{T})$ and $(S, \mathcal{B}(S))$ is a function $\delta: T \rightarrow \operatorname{Prob}(S)$ such that for every $B \in \mathcal{B}(S)$ the real-valued function $t \mapsto \delta(t)(B)$ is $\mathcal{T}$-measurable on $T$; see (Ash 1972, 2.6 or Neveu 1965 , III.2). Let $\mathcal{R}(T ; S)$ denote the set of all such transition probabili- 
ties. Clearly, such transition probabilities act as the natural mixed action profiles in a game-theoretical model (see Sect. 3).

Denote by $\mathcal{M}(T ; S)$ the set of all functions $f: T \rightarrow S$ that are measurable with respect to $(T, \mathcal{T})$ and $(S, \mathcal{B}(S))$. Such measurable functions will form the natural pure action profiles in Sect. 3. For any $f \in \mathcal{M}(T ; S)$ the corresponding degenerate (or Dirac) transition probability, denoted by $\epsilon_{f} \in \mathcal{R}(T ; S)$, is given by $\epsilon_{f}(t)(B):=1$ if $f(t) \in B$ and $\epsilon_{f}(t):=0$ if $f(t) \notin B$. As is well-known [see (Ash 1972, 2.6.2 or Neveu 1965, III.2)], together with the "starting measure" $\mu$, any transition probability $\delta$ in $\mathcal{R}(T ; S)$ induces a product measure $\pi_{\delta}:=\mu \otimes \delta$ on the product space $(T \times S, \mathcal{T} \otimes \mathcal{B}(S))$; it is determined by

$$
\pi_{\delta}(E \times B):=\int_{E} \delta(t)(B) \mu(d t), \quad E \in \mathcal{T}, \quad B \in \mathcal{B}(S) .
$$

Thus, for any $\mathcal{T} \otimes \mathcal{B}(S)$-measurable function $g: T \times S \rightarrow[0,+\infty]$ one can define the integral expression

$$
I_{g}(\delta):=\int_{T \times S} g d \pi_{\delta}=\int_{T}\left[\int_{S} g(t, s) \delta(t)(d s)\right] \mu(d t),
$$

with the usual extension $I_{g}(\delta):=I_{g^{+}}(\delta)-I_{g^{-}}(\delta)$ in case either $g^{+}:=\max (0, g)$ or $g^{-}:=\max (0,-g)$ is $\pi_{\delta}$-summable. See (Neveu 1965, III.2) for details about (1). For a degenerate transition probability $\delta=\epsilon_{f}$, as defined above, (1) gives in particular

$$
I_{g}\left(\epsilon_{f}\right)=\int_{T} g(t, f(t)) \mu(d t)=: J_{g}(f) .
$$

Theorem 2.1 (Lyapunov's theorem for Young measures (Balder 2000, Theorem 5.10) For $d \in \mathbb{N}$ let $g:=\left(g_{1}, \ldots, g_{d}\right): T \times S \rightarrow \mathbb{R}^{d}$ be $\mathcal{T} \otimes \mathcal{B}(S)$-measurable and let $\delta \in \mathcal{R}(T ; S)$ be such that $I_{|g|}(\delta)<+\infty$, with $|g(t, s)|:=\left(\sum_{j}\left|g_{j}(t, s)\right|^{2}\right)^{1 / 2}$. Then there exists a function $f: T \rightarrow S$, measurable with respect to $(T, \mathcal{T})$ and $(S, \mathcal{B}(S))$, such that

(i) $I_{g_{j}}(\delta)=J_{g_{j}}(f)$ for $j=1, \ldots, d$,

(ii) $f(t) \in \operatorname{supp} \delta(t)$ for $\mu$-almost every $t$ in $T$.

Similar versions of part (i), which is stated in terms of so-called integral functionals, have already been known for a considerable amount of time; for instance, see Theorem 3 on (Berliocchi and Lasry 1973, p. 153) or Theorem 1' on (Balder 1984, p. 466). It should be pointed out that it is not hard to deduce part (ii) from part (i) by adding the characteristic function $g_{0}:=1_{G}$ of the graph $G$ of the multifunction $t \mapsto \operatorname{supp} \delta(t)$ to the collection $g_{1}, \ldots, g_{d}$. See also Remark 2.2. Viewed in this way, Theorem 2.1 can be said to be just a reformulation of Theorem 1' in Balder (1984) [or of (Berliocchi and Lasry, 1973, Theorem 3) if $\sum_{j} g_{j}(t, s)$ has an additional 
inf-compactness property in the variable $s$ - that property certainly holds when $S$ is a finite set], provided that the $g_{j}(t, \cdot)$ are in addition lower semicontinous, as they are automatically when $S$ is a finite set.

Remark 2.1 By definition, the $d$ identities in (i) of the above Lyapunov theorem for Young measures mean

$$
\int_{T}\left[\int_{S} g_{j}(t, s) \delta(t)(d s)\right] \mu(d t)=\int_{T} g_{j}(t, f(t)) \mu(d t), \quad j=1, \ldots, d .
$$

Observe that an apparently more general formulation would have finitely many sets $C_{1}, \ldots, C_{l}$ in $\mathcal{T}$, finitely many finite and non-atomic measures $\mu_{1}, \ldots, \mu_{m}$ on $(T, \mathcal{T})$ and the following identities:

$$
\begin{aligned}
\int_{C_{i}}\left[\int_{S} g_{j}(t, s) \delta(t)(d s)\right] \mu_{k}(d t)= & \int_{C_{i}} g_{j}(t, f(t)) \mu_{k}(d t), \\
& (i, j, k)=(1,1,1), \ldots,(l, d, m) .
\end{aligned}
$$

Such a formulation of part (i) is reminiscent of the original Dvoretzky-WaldWolfowitz purification device. However, observe that this kind of generalization of Theorem 2.1 is gratuitous, because it is already contained in Theorem 2.1 itself: indeed, the above formulation follows if we apply Theorem 2.1 to $g:=\left(g_{1,1,1}, \ldots, g_{l, d, m}\right)$, with $g_{i, j, k}(t, s):=1_{C_{i}}(t) g_{j}(t, s) \phi_{k}(t)$. Here $1_{C_{i}}$ is the characteristic function of $C_{i}$ and $\phi_{k}:=d \mu_{k} / d \mu$ stands for any fixed version of the Radon-Nikodym derivative of $\mu_{k}$ with respect to the sum measure $\mu:=\sum_{k=1}^{m} \mu_{k}$, which is also non-atomic. ${ }^{1}$

Remark 2.2 The above setup is slightly less general than in Balder (2002), where $S$ is merely a completely regular Suslin space. However, in Balder (2002) an instrumental metric $\rho$ on $S$ was introduced, thanks to its compactness conditions (Balder 2002, p. 440) (see also Balder 2000). The $\rho$-topology is weaker than the original, completely regular topology [hence, $(S, \rho)$ is metrizable Suslin] and yet it coincides with the latter on all compact subsets of $S$. The role played by this metric $\rho$ has been incorrectly represented in the statement of Lyapunov's theorem for Young measures in Balder (2000, 2002): complete regularity of the Suslin space $S$ is enough for its part (i), but metrizability of $S$ (via a metric such as $\rho$ ) is needed for its part (ii). That is because measurability of $G:=\{(t, s) \in T \times S: s \in \operatorname{supp} \delta(t)\}$ can only be ensured if $S$ is metrizable. Indeed, regardless of metrizability of $S$, the multifunction $t \mapsto \operatorname{supp} \delta(t)$ is measurable in the sense of (Castaing and Valadier 1977, III.10): for any open $O \subset S$ the set $\{t \in T: \operatorname{supp} \delta(t) \cap O \neq \emptyset\}$ equals $\{t \in T: \delta(t)(O) \neq 0\}$, which is $\mathcal{T}$-measurable. However, to conclude from this that $G$ is measurable with respect to $\mathcal{T} \otimes \mathcal{B}(S)$ requires that $S$ is metrizable (see Castaing and Valadier 1977, III.13).

\footnotetext{
1 Actually, any $\sigma$-finite measure is equivalent, in the sense of mutual absolute continuity, to a finite measure, so the above easily extends further to non-atomic measures $\mu_{k}$ that are $\sigma$-finite.
} 
The author is indebted to F. Martins-da-Rocha for pointing this out.

When $S$ is a finite set, Theorem 2.1 has the following specialization:

Corollary 2.1 Suppose that the set $S$ is finite. Let $g:=\left(g_{1}, \ldots, g_{d}\right): T \times S \rightarrow \mathbb{R}^{d}$ be such that $g(\cdot, s)$ is $\mu$-integrable for every $s \in S$ and let $\delta \in \mathcal{R}(T ; S)$. Then there exists a $\mathcal{T}$-measurable function $f: T \rightarrow S$ such that

(i) $I_{g_{j}}(\delta)=J_{g_{j}}(f)$ for $j=1, \ldots, d$,

(ii) $f(t) \in \operatorname{supp} \delta(t)=\{s \in S: \delta(t)(\{s\})>0\}$ for $\mu$-almost every $t$ in $T$,

(iii) $\left.\mu \otimes \delta\right|_{S}=\mu\left(f^{-1}(\cdot)\right)$.

Here $\left.\mu \otimes \delta\right|_{S}$ denotes the marginal measure $\pi_{\delta}(T \times \cdot)$, induced by $\pi_{\delta}=\mu \otimes \delta$ on $S$. This corollary follows from Theorem 2.1 by adding the finitely many characteristic functions $g_{\bar{s}}:(t, s) \mapsto 1_{\{\bar{s}\}}(s)$, with $\bar{s} \in S$, to the already given finitely many functions $g_{1}, \ldots, g_{d}$, because (1) immediately gives $I_{g_{\bar{s}}}(\delta)=\mu(T \times\{\bar{s}\})$ and $J_{g_{\bar{s}}}(f)=$ $\mu\left(f^{-1}(\bar{s})\right)$. From this we obtain $\mu(T \times B)=\mu\left(f^{-1}(B)\right)$ for every $B \subset \mathcal{B}(S)=2^{S}$ (observe that this is where finiteness of $S$ is used). By Remark 2.1 and Corollary 2.1 it is evident that Corollary 1 of Khan et al. (2006), claimed to be a result proven for the first time on (Khan et al. 2006, p. 3), is contained in Theorem 2.1 (see Theorem A.3 in the appendix). Similarly, it can be demonstrated that Theorem 2.1 [and also its precursor (Balder 1984, Theorem 1') — see also (Balder 1985)] extends the purification result of Dvoretzky et al. (1950). In Khan et al. (2006) it is also stated that a first but unproven version of such a result figures in Theorem 4 of Milgrom and Weber (1985); however, see our earlier comments regarding the earlier papers (Balder 1984; Berliocchi and Lasry 1973).

\section{A purification result from Balder (2002)}

Recall from Sect.1 that Step 2 of our introductory proof-scheme was executed in (Balder 2002, Step 4, p. 465), in the context of a game in internal-external form, by implementing the purification device Theorem 2.1 [the latter figures there as Theorem 4.2.3 in Balder (2002)]. The concept of a game in internal-external form stems from Balder (1995). For models with a measure space of players it would appear to be a quite natural development of the classical notion of a strategic form game. To realize this more keenly, it should be kept in mind that for non-discrete $T$ the mapping $(t, f) \mapsto(f(\tau))_{\tau \neq t}$ has very bad measurability properties; see (Balder 2000).

In this section we shall formulate, in a simplified version of the model presented in Balder (2002), this Step 4 in Balder (2002) in the form of an independent result about purification per se. Of course, for this we only need to adopt the measurability assumptions made in Sect. 2.2 of Balder (2002). That is to say, the topological assumptions made in Sect. 2.2 of Balder (2002), which serve to establish mixed equilibrium existence by means of a fixed point argument and which do not intervene in that Step 4, need not be checked explicitly in the present papers, except for Remark 3.2. As in Sect. 2, let $(T, \mathcal{T}, \mu)$ be a finite and non-atomic measure space; here it is the set of players or players' types (in the latter case interpretation may differ from what 
is presented below). To stay in touch with Balder (2002), we observe that the model presented here is a special case of the one considered there, because, in the notation of (Balder 2002, Assumption 2.2.3), the present paper only uses $\hat{T}:=T$ and $\bar{T}:=\varnothing$. Further, we observe that in this paper all social feasibility aspects of Balder (2002) have been left out (i.e., trivialized). Observe also that for the purification results in the present paper the measure space $(T, \mathcal{T}, \mu)$ does not have to be taken to be separable, because for purification per se one essentially considers a single (but arbitrary) mixed action profile; this single profile generates a countably generated sub- $\sigma$-algebra of $\mathcal{T}$, to which we can apply a trick introduced in (Castaing and Valadier 1977, p. 78)—see Remark 4.2 in Balder (1999) for the details. Just as in Sect. 2, we suppose that $S$ is a Borel-measurable subset of a complete separable metric space. In the terminology of Balder (1995, 2002), $S$ is the action universe. By Remark 2.2.1 (v) of Balder (2002), we do not need any additional vectorial structure for our action universe $S$.

For every $t \in T$ let $S_{t} \subset S$ be a non-empty action set such that $\Sigma: t \mapsto S_{t}$ has a measurable graph; the latter is denoted by gph $\Sigma:=\left\{(t, s) \in T \times S: s \in S_{t}\right\}$. This is the measurability part (iii) of Assumption 2.2.3 in Balder (2002). Just as in Balder (2002), by $\mathcal{R}_{\Sigma}$ we denote the set of all $\delta \in \mathcal{R}(T ; S)$ with $\delta(t)\left(S_{t}\right)=1$ for $\mu$-a.e. $t$ in $T$ and by $\mathcal{S}_{\Sigma}$ the set of all $f \in \mathcal{M}(T ; S)$ with $f(t) \in S_{t}$ for $\mu$-a.e. $t$ in $T$. The set $\mathcal{R}_{\Sigma}$ constitutes the collection of all feasible mixed action profiles for our game model. Likewise, $\mathcal{S}_{\Sigma}$ forms the set of all feasible pure action profiles. Observe that the non-emptiness of $\mathcal{S}_{\Sigma}$ (whence of $\mathcal{R}_{\Sigma}$ ) is ensured by the von Neumann-Aumann measurable selection theorem (Castaing and Valadier 1977, III.22).

Let $m \in \mathbb{N}$. For every $t \in T$ let $U_{t}: S_{t} \times \mathbb{R}^{m} \rightarrow[-\infty,+\infty]$ be player $t$ 's payoff function. Following (Balder 1995), we could also restrict ourselves to a suitably chosen Borel-measurable subset $Y \subset \mathbb{R}^{m}$ and consider $U_{t}: S_{t} \times Y \rightarrow[-\infty,+\infty]$, but in this section we shall not implement this extension, because it is rather obvioussee Sects. 4.2 and 4.3 for such implementations. We suppose that for every $y \in \mathbb{R}^{m}$ the function $(t, s) \rightarrow U_{t}(s, y)$ is measurable with respect to the trace $\sigma$-algebra $(\mathcal{T} \otimes \mathcal{B}(S)) \cap$ gph $\Sigma$; note that this is the measurability part (ii) of Assumption 2.2.6 in Balder (2002). We shall say that the payoffs are integrably bounded on gph $\Sigma$ if for every $y \in \mathbb{R}^{m}$ there exists a $\mu$-integrable $\psi_{y}: T \rightarrow \mathbb{R}$ with $\left|U_{t}(s, y)\right| \leq \psi_{y}(t)$ for all $t \in T$ and $s \in S_{t}$. This condition is not needed for all results below-it will be stated explicitly when we need it. Evidently, when the set $S$ is finite and when $S_{t}=S$ for all $t$, as will be the case in Sect. 4, such integrable boundedness on gph $\Sigma$ amounts to $t \mapsto U_{t}(s, y)$ being $\mu$-integrable for every $s \in S$ and $y \in \mathbb{R}^{m}$. Further, let $g_{1}, \ldots, g_{m}:$ gph $\Sigma \rightarrow \mathbb{R}$ be measurable with respect to $(\mathcal{T} \otimes \mathcal{B}(S)) \cap$ gph $\Sigma$ and such that for every $i=1, \ldots, m$ there exists a $\mu$-integrable $\psi_{i}: T \rightarrow \mathbb{R}$ with $\left|g_{i}(t, s)\right| \leq \psi_{i}(t)$ for all $t \in T$ and $s \in S_{t}$; this is the measurability/integrability part of Assumption 2.2.4 in Balder (2002) and all remaining measurability assumptions in Balder (2002) hold for obvious or trivial reasons. It is evident that any $(\mathcal{T} \otimes \mathcal{B}(S)) \cap$ gph $\Sigma$-measurable function $g:$ gph $\Sigma \rightarrow \mathbb{R}$ can be extended automatically to a $\mathcal{T} \otimes \mathcal{B}(S)$-measurable function $\hat{g}: T \times S \rightarrow \mathbb{R}$ by setting

$$
\hat{g}:=\left\{\begin{array}{l}
g \text { on gph } \Sigma, \\
0 \text { on }(T \times S) \backslash \text { gph } \Sigma
\end{array}\right.
$$


We define the mixed and pure externality mappings $e: \mathcal{R}_{\Sigma} \rightarrow \mathbb{R}^{m}$ and $d: \mathcal{S}_{\Sigma} \rightarrow \mathbb{R}^{m}$ by

$$
e(\delta):=\left(I_{g_{j}}(\delta)\right)_{j=1}^{m}, d(f):=e\left(\epsilon_{f}\right)=\left(J_{g_{j}}(f)\right)_{j=1}^{m} .
$$

In this general model player $t$ 's expected payoff under the feasible mixed action profile $\delta \in \mathcal{R}_{\Sigma}$ is understood to be $\int_{S_{t}} U_{t}(s, e(\delta)) \delta(t)(d s)$; likewise, his/her payoff under the pure action profile $f \in \mathcal{S}_{\Sigma}$ is $U_{t}(f(t), d(f))$. In the present paper certain relevant comments in Balder $(2000,2002)$ about proper modeling in continuum games come down to the following. Each fixed player $t$ forms a singleton, so, rather roughly, he/she can be said to have $\mu$-measure zero. Therefore, under a given profile $\delta$, any deviations from the assigned mixed action $\delta(t)$ cannot influence the vector $e(\delta)$ that is used in this paper, because each component of $e(\delta)$ is evaluated as an integral over $(T, \mathcal{T}, \mu)$.

We shall say that the pure action profile $f \in \mathcal{S}_{\Sigma}$ is an internal-external-stable purification of $\delta \in \mathcal{R}_{\Sigma}$ if

(i) $d(f)=e(\delta)$ (external stability),

(ii) $f(t) \in \operatorname{supp} \delta(t)$ for $\mu$-a.e. $t$ in $T$ (internal stability).

This notion received no identifying name in Balder (2002), but it clearly functions there on p. 465. External stability means that for each player in the non-atomic measure space $T$ the influence of the totality of his/her opponents ("externality") does not change if the mixed action profile $\delta$ is changed into $\epsilon_{f}$. Similarly, internal stability indicates that any player $t$ 's own action under the pure action profile $f$ can maintain any desirable level of payoff that is already achieved by his/her own mixed action under the profile $\delta$. In this model a pure Cournot-Nash equilibrium profile is defined as a pure action profile $f \in \mathcal{S}_{\Sigma}$ such that

$$
f(t) \in \operatorname{argmax}_{s \in S_{t}} U_{t}(s, d(f)) \text { for } \mu \text {-a.e. } t \text { in } T \text {. }
$$

Also, a mixed Cournot-Nash equilibrium profile is defined to be a mixed action profile $\delta \in \mathcal{R}_{\Sigma}$ such that

$$
\delta(t)\left(\operatorname{argmax}_{s \in S_{t}} U_{t}(s, e(\delta))\right)=1 \text { for } \mu \text {-a.e. } t \text { in } T \text {. }
$$

See (Balder 1995) for more motivating details. The following properties of internalexternal stable purifications, although elementary, will turn out to be meaningful.

Remark 3.1 Suppose that $f \in \mathcal{S}_{\Sigma}$ is an internal-external-stable purification of $\delta \in$ $\mathcal{R}_{\Sigma}$. Then the following hold:

(i) $U_{t}(s, d(f))=U_{t}(s, e(\delta))$ for every $t \in T$ and $s \in S_{t}$.

(ii) If $\delta$ is a mixed Cournot-Nash equilibrium profile, then $f$ is a pure Cournot-Nash equilibrium profile.

(iii) $f$ can always be selected in such a way that aggregate payoff is conserved:

$$
\int_{T} U_{t}(f(t), d(f)) \mu(d t)=\int_{T}\left[\int_{S} U_{t}(s, e(\delta)) \delta(t)(d s)\right] \mu(d t),
$$


provided that the payoffs are integrably bounded on gph $\Sigma$ in the sense defined above.

Here (i), (ii) are immediate and (iii) follows by applying Remark 2.1 to an extension, in the sense of (2), of the function $(t, s) \rightarrow U_{t}(s, e(\delta))$. If so desired, one could also guarantee additional (iii) - like identities for related functions, as long as there are only finitely many of them. Thus, for instance, in a proof in (Balder 1995, p. 91) the above identity is used, but with $\arctan U_{t}$ instead of $U_{t}$ to remove any needs for integrable boundedness. Below, in Corollary 3.1, we shall encounter another instance of this.

With these definitions from Balder $(1995,2002)$ and the additional new terminology in place, the following purification result is as contained in Step 4 on (Balder 2002, p. 465).

Theorem 3.1 Every mixed action profile in $\mathcal{R}_{\Sigma}$ has an internal-external-stable purification in $\mathcal{S}_{\Sigma}$.

Even without any consultation of Balder (2002) this is very easy to deduce from Theorem 2.1: just extend the $g_{j}$ of the game model as in (2) to all of $T \times S$. Then apply Theorem 2.1 to any $\delta \in \mathcal{R}_{\Sigma}$; its part (i) takes care of external stability of the purification $f \in \mathcal{M}(T ; S)$ and its part (ii) ensures feasibility of that $f$ as well as internal stability of the purification $f$. The above theorem directly implies the following result; its part a follows by Remark 3.1 and its subsequent comments, and its part b follows by invoking Corollary 2.1 .

Corollary 3.1 a. Let $\left\{C_{1}, \ldots, C_{l}\right\}$ be a finite collection in $\mathcal{T}$. To every mixed action profile $\delta \in \mathcal{R}_{\Sigma}$ there corresponds $f \in \mathcal{S}_{\Sigma}$ such that

(i) $\quad d(f)=e(\delta)$ and in particular $U_{t}(s, d(f))=U_{t}(s, e(\delta))$ for every $t \in T$ and $s \in S_{t}$,

(ii) $f(t) \in \operatorname{supp} \delta(t)$ for $\mu$-a.e. $t$ in $T$.

(iii) for $i=1, \ldots, l$

$$
\int_{C_{i}} U_{t}(f(t), d(f)) \mu(d t)=\int_{C_{i}}\left[\int_{S} U_{t}(s, e(\delta)) \delta(t)(d s)\right] \mu(d t),
$$

provided that the payoffs are integrably bounded on gph $\Sigma$,

$b$. If in part a $S$ is in addition supposed to be a finite set, then $f$ can be chosen in such a way that, next to (i)-(iii), one also has

(iv) $\left.\mu \otimes \delta\right|_{S}=\mu\left(f^{-1}(\cdot)\right)$.

Remark 3.2 As in part b of the above corollary, suppose that the action universe $S$ is finite for every $t \in T$. Then, as an additional bonus, Theorem 2.2.1 of Balder (2002), of which an appropriate simplification has been stated in Theorem A.5 in the appendix, gives that there exists a pure Cournot-Nash equilibrium profile for the above game, provided that the following continuity condition holds: $U_{t}(s, \cdot)$ is continuous on $\mathbb{R}^{m}$ for every $t \in T$ and $s \in S_{t}$. Indeed, by the finiteness of $S$ this causes the three conditions that are stated just before Theorem A.5 to be fulfilled. 


\section{Applications to four game models in Khan et al. (2006)}

In this section we shall show that each of the four finite action game models in Sects. 36 of Khan et al. (2006) can be reformulated in the internal-external form of the previous section, so that Corollary 3.1 applies to it. It will also be shown that, as a consequence, Theorems 1-4 in Khan et al. (2006) (or improvements of those results) follow from Corollary 3.1. Each of the following three subsections deals with such a reformulation; for the reader's benefit, the first reformulation is extensive, because it would appear that it has not been presented before in the literature. The second and third subsections are shorter, because the pertinent reformulations are more direct and well-known (Balder 1991, 1995).

\subsection{Games with incomplete information in Milgrom-Weber's sense}

We begin by describing a model introduced by Milgrom and Weber (1985), as extended by the present author Balder (1988) to measurable type spaces. This model is as used in Sect. 3 of Khan et al. (2006), although our notation is a little different.

Let $I$ be a set of $l$ players $1, \ldots, l$; each player $i$ has a finite action set $A_{i}$ and a measurable space $\left(\Omega_{i}, \mathcal{F}_{i}\right)$ of types. Let $\Omega_{0}:=\left\{\omega_{01}, \ldots, \omega_{0 m}\right\}$ be a finite set of states of nature (commonly observable). Also, let $A$ be the Cartesian product $\Pi_{i \in I} A_{i}$. Since $A$ is finite, it can be represented as $A:=\left\{a^{1}, \ldots, a^{p}\right\}$, where each $a^{q}$ is a vector: $a^{q}=\left(a_{1}^{q}, \ldots, a_{l}^{q}\right)$. Likewise, we define $\Omega:=\Pi_{i \in I} \Omega_{i}$. This space is equipped with the product $\sigma$-algebra $\mathcal{F}:=\otimes_{i \in I} \mathcal{F}_{i}$. Let $\eta$ be a given probability measure on $\left(\Omega_{0} \times \Omega, \mathcal{F}_{0} \otimes \mathcal{F}\right)$. We denote by $\eta\left(\cdot ; \omega_{0 k}\right)$ the conditional probability on $(\Omega, \mathcal{F})$ under $\eta$, given that the state $\omega_{0 k} \in \Omega_{0}$ has been realized. Thus, we have $\eta\left(\cdot ; \omega_{0 k}\right):=$ $\eta\left(\left\{\omega_{0 k}\right\} \times \cdot\right) / \mu_{0 k}$, where $\mu_{0 k}:=\eta\left(\left\{\omega_{0 k}\right\} \times \Omega\right)$ is the probability of the state $\omega_{0 k}$ (we can suppose without loss of generality that each $\mu_{0 k}$ is strictly positive or else remove the corresponding $\omega_{0 k}$ from the set $\Omega_{0}$ ). We suppose that the following conditional independence condition from Milgrom and Weber (1985) is met: for every $k, 1 \leq k \leq m$, the conditional probability $\eta\left(\cdot ; \omega_{0 k}\right)$ is equal to the product $\mu^{k}:=\times_{i \in I} \mu_{i}^{k}$ of its marginal probabilities $\mu_{i}^{k}:=\eta_{i}\left(\cdot \times \Omega_{-i} ; \omega_{0 k}\right)$ on the respective type spaces $\left(\Omega_{i}, \mathcal{F}_{i}\right)$. Here $\Omega_{-i}:=\Pi_{j \in I, j \neq i} \Omega_{j}$ follows the standard abusive notation in game theory that will be applied frequently. Thus, we are also free to write $a^{q}=\left(a_{i}^{q}, a_{-i}^{q}\right)$, etc. We shall suppose that $\mu_{i}^{k}$ is non-atomic for every $k, 1 \leq k \leq m$ and $i \in I$.

In this model a mixed strategy for player $i \in I$ is a transition probability $\delta_{i} \in$ $\mathcal{R}\left(\Omega_{i} ; A_{i}\right)$; see section 2 for this definition. Of course, then a pure strategy for her/him is a function $f_{i} \in \mathcal{M}\left(\Omega_{i} ; A_{i}\right)$, that is to say, a function measurable with respect to $\left(\Omega_{i}, \mathcal{F}_{i}\right)$ and $\left(A_{i}, \mathcal{B}\left(A_{i}\right)\right)$. The vectors in $\Pi_{i \in I} \mathcal{R}\left(\Omega_{i} ; A_{i}\right)$ will be called mixed strategy profiles; similarly, $\Pi_{i \in I} \mathcal{M}\left(\Omega_{i} ; A_{i}\right)$ is the set of all pure strategy profiles. Let $u_{i}: A \times \Omega_{0} \times \Omega_{i} \rightarrow \mathbb{R}$ be the payoff function of player $i \in I$; for every $q, 1 \leq q \leq p$, and $k, 1 \leq k \leq m$, the function $u_{i}\left(a^{q}, \omega_{0 k}, \cdot\right)$ is supposed to be $\mathcal{F}_{i}$-measurable and integrable with respect to the marginal probability $\eta\left(\Omega_{0} \times \cdot \times \Omega_{-i}\right)=\sum_{k=1}^{m} \mu_{0 k} \mu_{i}^{k}$ on $\left(\Omega_{i}, \mathcal{F}_{i}\right)$. Thus, for any mixed strategy profile $\left(\delta_{1}, \ldots, \delta_{l}\right)$ the Bayesian-expected payoff for player $i \in I$, given by 


$$
\begin{aligned}
P_{i}\left(\delta_{1}, \ldots, \delta_{l}\right):= & \sum_{k=1}^{m} \mu_{0 k} \sum_{q=1}^{p} \int_{\Omega} u_{i}\left(a^{q}, \omega_{0 k}, \omega_{i}\right) \delta_{i}\left(\omega_{i}\right)\left(\left\{a_{i}^{q}\right\}\right) \Pi_{j \in I, j \neq i} \delta_{j}\left(\omega_{j}\right) \\
& \left(\left\{a_{j}^{q}\right\}\right) \mu^{k}(d \omega) \\
= & \sum_{k=1}^{m} \mu_{0 k} \sum_{q=1}^{p} \Pi_{j \in I, j \neq i}\left(\mu_{j}^{k} \otimes \delta_{j}\right)\left(\Omega_{j} \times\left\{a_{j}^{q}\right\}\right) \\
& \int_{\Omega_{i}} u_{i}\left(a^{q}, \omega_{0 k}, \omega_{i}\right) \delta_{i}\left(\omega_{i}\right)\left(\left\{a_{i}^{q}\right\}\right) \mu_{i}^{k}\left(d \omega_{i}\right),
\end{aligned}
$$

is well-defined.

We shall now reformulate the above Milgrom-Weber type game as a game that is in internal-external form. To begin with, we set $T:=\cup_{i \in I} \Omega_{i}$ (this is the "type universe") and we suppose without loss of generality that $T$ is the disjoint union of the sets $\Omega_{i}$ (or else we should work with $\hat{\Omega}_{i}:=\Omega_{i} \times\{i\}, i \in I$ ). Thus, for every $t \in T$ there is a unique index $i(t) \in I$ with $t \in \Omega_{i(t)}$. The $\sigma$-algebra $\mathcal{T}$ is defined on $T$ in the obvious way: $E \in \mathcal{T}$ if and only if $E \cap \Omega_{i}$ belongs to $\mathcal{F}_{i}$ for every $i \in I$; this causes the restriction of $(T, \mathcal{T})$ to $\left(\Omega_{i}, \mathcal{F}_{i}\right)$ to be measurable. Also $t \mapsto i(t)$ is then $\mathcal{T}$-measurable. For each $i \in I$ let $\mu_{i}$ be the measure on $\left(\Omega_{i}, \mathcal{F}_{i}\right)$ that is given by $\mu_{i}:=\sum_{k=1}^{m} \mu_{0 k} \mu_{i}^{k}$. To define the measure $\mu$ on $(T, \mathcal{T})$ we concatenate the measures $\mu_{i}$; more precisely, we set $\mu(E):=\sum_{i \in I} \mu_{i}\left(E \cap \Omega_{i}\right)$ for any $E \in \mathcal{T}$. This defines a finite non-atomic measure $\mu$.

Further, as our action universe we use $S:=\cup_{i \in I} A_{i}$; of course, here it is a finite set. For $t \in T$ we define $\Sigma(t):=S_{t}:=A_{i(t)}$ (in other words, $S_{t}:=\Omega_{j}$ if $i(t)=j$ ). This gives gph $\Sigma=\cup_{i \in I} \Omega_{i} \times A_{i}$. To a mixed strategy profile $\left(\delta_{1}, \ldots, \delta_{l}\right)$ in $\Pi_{i \in I} \mathcal{R}\left(\Omega_{i} ; A_{i}\right)$ there corresponds obviously, by concatenation, an element $\delta$ of $\mathcal{R}_{\Sigma}$ : namely, we can define

$$
\delta(t):=\delta_{i}(t) \text { if } t \in \Omega_{i}, \quad i \in I
$$

In a more condensed style, which we shall not follow, this could equivalently be stated as $\delta(t):=\delta_{i(t)}(t)$, or even as $\delta:=\delta_{i(\cdot)}$. Instead, we prefer to denote this concatenated transition probability by $\delta=\delta_{1} \smile \cdots \smile \delta_{l}$. Conversely, because of the above form of gph $\Sigma$, it is immediately seen that by restricting an action profile $\delta \in \mathcal{R}_{\Sigma}$ to the respective subsets $\Omega_{i}$, one obtains the profile $\left(\delta_{1}, \ldots, \delta_{l}\right)$, with $\delta_{i} \in \mathcal{R}\left(\Omega_{i} ; A_{i}\right)$ given by

$$
\delta_{i}(t):=\delta(t) \quad \text { if } t \in \Omega_{i}
$$

for any $i \in I$. We shall denote the latter by $\delta_{i}:=\left.\delta\right|_{\Omega_{i}}$. In tandem, (5)-(6) allow us to identify feasible mixed action profiles (in the sense of Sect. 3) with mixed strategy profiles for the above Milgrom-Weber type game. In a completely similar way the above considerations allow us to identify feasible pure action profiles with pure strategy profiles. 
Next, define $g_{i, q, k}:$ gph $\Sigma \rightarrow \mathbb{R}$ as follows:

$$
g_{i, q, k}(t, s):= \begin{cases}1_{\left\{a_{i}^{q}\right\}}(s) \phi_{i}^{k}(t) & \text { if } t \in \Omega_{i} \\ 0 & \text { if } t \in T \backslash \Omega_{i}\end{cases}
$$

Here $\phi_{i}^{k}$ stands for a fixed version of the Radon-Nikodym derivative $d \mu_{i}^{k} / d \mu_{i}$ on $\Omega_{i}$. As our mixed and pure externality mappings we choose

$$
e(\delta):=\left(I_{g_{1,1,1}}(\delta), \ldots, I_{g_{l, p, m}}(\delta)\right), \quad d(f):=e\left(\epsilon_{f}\right)=\left(J_{g_{1,1,1}}(f), \ldots, J_{g_{l, p, m}}(f)\right) .
$$

By (1) we have

$$
\begin{aligned}
e_{i, q, k}(\delta):=I_{g_{i, q, k}}(\delta) & :=\int_{T}\left[\int_{S} g_{i, q, k}(t, s) \delta(t)(d s)\right] \mu(d t) \\
& =\int_{\Omega_{i}} \delta_{i}\left(\omega_{i}\right)\left(\left\{a_{i}^{q}\right\}\right) \phi_{i}^{k}\left(\omega_{i}\right) \mu_{i}\left(d \omega_{i}\right)=\left(\mu_{i}^{k} \otimes \delta_{i}\right)\left(\Omega_{i} \times\left\{a_{i}^{q}\right\}\right) .
\end{aligned}
$$

Therefore, our previous expression for the Bayesian-expected payoff gives

$$
\begin{aligned}
P_{i}\left(\delta_{1}, \ldots, \delta_{l}\right)= & \sum_{k=1}^{m} \mu_{0 k} \sum_{q=1}^{p} \Pi_{j \in I, j \neq i} e_{j, q, k}(\delta) \\
& \int_{\Omega_{i}} u_{i}\left(a_{i}^{q}, a_{-i}^{q}, \omega_{0 k}, \omega_{i}\right) \delta_{i}\left(\omega_{i}\right)\left(\left\{a_{i}^{q}\right\}\right) \mu_{i}^{k}\left(d \omega_{i}\right) .
\end{aligned}
$$

This suggests the following internal-external reformulation of the payoff structure: for $t \in T, s \in S_{t}$ and $\left(y_{1,1,1}, \ldots, y_{l, p, m}\right) \in \mathbb{R}^{l p m}$ define

$$
U_{t}\left(s, y_{1,1,1}, \ldots, y_{l, p, m}\right):=\sum_{k=1}^{m} \mu_{0 k} \sum_{q=1}^{p} \Pi_{j \in I, j \neq i} y_{j, q, k} u_{i}\left(s, a_{-i}^{q}, \omega_{0 k}, t\right) \phi_{i}^{k}(t)
$$

where $i:=i(t)$, whence $t \in \Omega_{i}$ and $S_{t}=A_{i}$, so the definition makes sense. It is easy to see that in this way the payoffs are integrably bounded on gph $\Sigma=\cup_{i} \Omega_{i} \times A_{i}$ (note that $S:=\cup_{i} A_{i}$ is finite and recall our comment in Sect.3 about integrable boundedness in that special situation). For the above mixed externality mapping we now have that for every $t \in T, s \in S_{t}$, with $i:=i(t)$,

$$
U_{t}(s, e(\delta))=\sum_{k=1}^{m} \mu_{0 k} \sum_{q=1}^{p} \Pi_{j \in I, j \neq i} e_{j, q, k}(\delta) u_{i}\left(s, a_{-i}^{q}, \omega_{0 k}, t\right) \phi_{i}^{k}(t)
$$


Therefore, it follows that for $i=1, \ldots, l$

$$
P_{i}\left(\delta_{1}, \ldots, \delta_{l}\right)=\int_{\Omega_{i}}\left[\int_{S_{t}} U_{t}(s, e(\delta)) \delta(t)(d s)\right] \mu(d t),
$$

because the restriction of $\mu$ to the integration domain $\Omega_{i}$ equals $\mu_{i}$ and because the restriction of $\delta$ to $\Omega_{i}$ is $\delta_{i}$, with $S_{t}=A_{i}$ for all $t \in \Omega_{i}$. As before, here (5)-(6) connect $\left(\delta_{1}, \ldots, \delta_{l}\right)$ on the left with its concatenation $\delta=\delta_{1} \smile \cdots \smile \delta_{l}$ on the right. We are now in a position to apply Corollary 3.1, using $C_{i}:=\Omega_{i}$. By the above substitutions this gives:

Theorem 4.1 For every mixed strategy profile $\left(\delta_{1}, \ldots, \delta_{l}\right)$ there exists a pure strategy profile $\left(f_{1}, \ldots, f_{l}\right)$ with the following properties: for every $i \in I$

(i) $f_{i}\left(\omega_{i}\right) \in \operatorname{supp} \delta_{i}\left(\omega_{i}\right)$ for $\mu_{i}$-a.e. $\omega_{i}$ in $\Omega_{i}$,

(ii) $\left(\mu_{i}^{k} \otimes \delta_{i}\right)\left(\Omega_{i} \times \cdot\right)=\mu_{i}^{k}\left(f_{i}^{-1}(\cdot)\right)$ for every $1 \leq k \leq m$ and in particular

$$
\begin{array}{r}
\sum_{k=1}^{m} \mu_{0 k} \sum_{q=1}^{p} \Pi_{j \in I, j \neq i}\left(\mu_{j}^{k} \otimes \delta_{j}\right)\left(\Omega_{j} \times\left\{a_{j}^{q}\right\}\right) u_{i}\left(a_{i}, a_{-i}^{q}, \omega_{0 k}, \omega_{i}\right) \phi_{i}^{k}\left(\omega_{i}\right) \\
=\sum_{k=1}^{m} \mu_{0 k} \sum_{q=1}^{p} \Pi_{j \in I, j \neq i} \mu_{j}^{k}\left(f_{j}^{-1}\left(\left\{a_{j}^{q}\right\}\right)\right) u_{i}\left(a_{i}, a_{-i}^{q}, \omega_{0 k}, t\right) \phi_{i}^{k}\left(\omega_{i}\right),
\end{array}
$$

$$
\text { for every } \omega_{i} \in \Omega_{i} \text { and } a_{i} \in A_{i}
$$

(iii) $P_{i}\left(\delta_{1}, \ldots, \delta_{l}\right)=P_{i}\left(\epsilon_{f_{1}}, \ldots, \epsilon_{f_{l}}\right)$.

The following remark shows that (iii) above implies the so-called strong payoff equivalence property in (Khan et al. 2006, Definition 1):

Remark 4.1 Because of (8), Theorem 4.1(ii) implies that for every $i \in I$

$$
\begin{gathered}
P_{i}\left(\delta_{1}, \ldots, \delta_{i-1}, \delta_{i}^{\prime}, \delta_{i+1}, \ldots, \delta_{l}\right)=P_{i}\left(\epsilon_{f_{1}}, \ldots, \epsilon_{f_{i-1}}, \delta_{i}^{\prime}, \epsilon_{f_{i+1}}, \ldots, \epsilon_{f_{l}}\right) \\
\text { for every } \delta_{i}^{\prime} \in \mathcal{R}\left(\Omega_{i} ; A_{i}\right) .
\end{gathered}
$$

As a consequence, Theorem 4.1 implies Theorem 1 of Khan et al. (2006).

Remark 4.2 Given its proof in (Khan et al. 2006, p. 99-100), Theorem 1 of Khan et al. (2006) is identical to Theorem 4.1. However, we can go a little further (more extensions are indicated following this remark). One rather direct extension of the model in this subsection is obtained by allowing for the following payoffs that are a little more general: allow, instead of the above $u_{i}: A \times \Omega_{0} \times \Omega_{i} \rightarrow \mathbb{R}$, payoffs $\hat{u}_{i}: A \times \Omega_{0} \times \Omega \rightarrow \mathbb{R}$ that have the following form

$$
\hat{u}_{i}\left(a^{q}, \omega_{0 k}, \omega_{1}, \ldots, \omega_{l}\right)=u_{i}\left(a^{q}, \omega_{0 k}, \omega_{i}\right) \Pi_{j \in I} \chi_{j}^{k, q}\left(\omega_{j}\right),
$$


where each function $\chi_{i}^{k, q}: \Omega_{i} \rightarrow \mathbb{R}_{+}$is $\mu_{i}^{k}$-integrable. To see that this is possible, just "absorb" the new factors $\chi_{i}^{k, q}$ by defining $\mu_{i}^{k, q}$ via $\mu_{i}^{k, q}(E):=\int_{E} \chi_{i}^{k, q} d \mu_{i}^{k}$ and replace $g_{i, q, k}$ in the above proof by

$$
g_{i, q, k}(t, s):= \begin{cases}1_{\left\{a_{i}^{q}\right\}}(s) \phi_{i}^{k, q}(t) & \text { if } t \in \Omega_{i}, \\ 0 & \text { if } t \in T \backslash \Omega_{i} .\end{cases}
$$

Here $\phi_{i}^{k, q}\left(\omega_{i}\right):=\chi_{i}^{k, q}\left(\omega_{i}\right) \phi_{i}^{k}\left(\omega_{i}\right)$ is a fixed version of the Radon-Nikodym derivative $d \mu_{i}^{k, q} / d \mu_{i}$. This leads to an extension of Theorem 4.1. The remaining details are left to the reader.

Incidentally, it also follows by (8) that any Cournot-Nash equilibrium profile [mixed or pure] in the sense of Sect. 3 can be identified with a Bayesian-Nash equilibrium strategy profile [mixed or pure] in the present Milgrom-Weber type game (of course, the vice versa reversal of this statement is also true). As already mentioned in Remark 3.2, a major advantage of our approach is that, at the same time, we can also provide a pure equilibrium existence result for the Milgrom-Weber type game, because of the above reformulation of that game. Such existence is not discussed at all in Theorems 1 and 2 of Khan et al. (2006). By including other features of Theorem 2.2.1 in Balder (2002), omitted here (e.g., inclusion of social feasibility) we could have pushed these results (both for purification per se and existence) a little further. As observed in Khan et al. (2006), a model à la Radner and Rosenthal (1982), as studied in Sect. 4 of Khan et al. (2006), is a special case of the previous Milgrom-Weber model because it works with a singleton set $\Omega_{0}$. For this reason it is evident that Theorem 2 of Khan et al. (2006) follows from Corollary 3.1 and we shall not explicitly show it.

\subsection{Non-atomic games in Schmeidler's sense}

Schmeidler's model of a continuum game Schmeidler (1973), in the special form treated in Sect. 5 of Khan et al. (2006), can be described by making some direct substitutions in the internal-external game model of Sect.3. Our present discussion will be slightly more general than in Khan et al. (2006), to bring out the strong purification aspect of an arbitrary mixed strategy (Sects. 5 and 6 in Khan et al. (2006) do not address purification of arbitrary mixed strategies, as do its Sects. 3 and 4, but merely purification of mixed equilibrium strategies). Let $A$ be a finite set of actions. Let $C \in \mathcal{T}$ be fixed, with $\mu(C)>0$ (in Khan et al. (2006) one has $C=T$ and $\mu(T)=1$ ). One should think of $C$ as the set of players who are, in a sense, the only essential players in the game (i.e., actions chosen by "dummy players" in $T \backslash C$ do not influence any of the players). For $t \in T$ let $u_{t}: A \times \operatorname{Prob}(A) \rightarrow[-\infty,+\infty]$ be player $t$ 's payoff function. We suppose that $t \mapsto u_{t}(a, v)$ is $\mathcal{T}$-measurable for every $a \in A$ and $v \in \operatorname{Prob}(A)$. Then

$$
\Pi_{t}(\delta):=\sum_{a \in A} u_{t}(a,(\mu \otimes \delta)(C \times \cdot) / \mu(C)) \delta(t)(\{a\})
$$


defines player $t$ 's expected payoff under the mixed action profile $\delta$. Corollary 3.1 implies the following result, which extends Theorem 3 of Khan et al. (2006).

Theorem 4.2 For every mixed strategy profile $\delta \in \mathcal{R}(T ; A)$ there exists a pure strategy profile $f \in \mathcal{M}(T ; A)$ with the following properties:

(i) $\mu\left(C \cap f^{-1}(\cdot)\right) / \mu(C)=(\mu \otimes \delta)(C \times \cdot) / \mu(C)$ and in particular

$$
u_{t}\left(a, \mu\left(C \cap f^{-1}(\cdot)\right) / \mu(C)\right)=u_{t}(a, \mu \otimes \delta(C \times \cdot) / \mu(C))
$$

for every $t \in T$ and $a \in A$,

(ii) $f(t) \in \operatorname{supp} \delta(t)$ for $\mu$-a.e. $t$ in $T$,

(iii) $\int_{C} \Pi_{t}(\delta) \mu(d t)=\int_{C} \Pi_{t}\left(\epsilon_{f}\right) \mu(d t)$ and $\int_{T \backslash C} \Pi_{t}(\delta) \mu(d t)=\int_{T \backslash C} \Pi_{t}\left(\epsilon_{f}\right) \mu(d t)$, provided that $t \mapsto u_{t}(a, v)$ is $\mu$-integrable for every $v \in \operatorname{Prob}(A)$.

(iv) for every $\delta^{\prime} \in \mathcal{R}(C ; A)$ and $t \in T$

$$
\Pi_{t}\left(\left.\epsilon_{f}\right|_{T \backslash C} \smile \delta^{\prime}\right)=\Pi_{t}\left(\left.\delta\right|_{T \backslash C} \smile \delta^{\prime}\right) .
$$

In (iv) we denote concatenations in the way introduced in Sect. 4.1 and (iii) states that aggregate payoff is conserved by both $C$ and $T \backslash C$.

We apply Corollary 3.1 by making the following substitutions: we set $S:=A$ and $S_{t}:=A$ for all $t \in T$. Also, for any $v \in \operatorname{Prob}(A)$, we define $\Phi(v) \in \mathbb{R}^{m}$ by $\Phi(v):=\left(v\left(\left\{a^{1}\right\}\right), \ldots, v\left(\left\{a^{m}\right\}\right)\right)$. Then $Y:=\Phi(\operatorname{Prob}(A))$, the image of $\operatorname{Prob}(A)$ under $\Phi$, is precisely the unit simplex in $\mathbb{R}^{m}$ and $\Phi: \operatorname{Prob}(A) \rightarrow Y$ is a (continuous) bijection. We define $U_{t}: A \times \mathbb{R}^{m} \rightarrow[-\infty,+\infty]$ by

$$
U_{t}(a, y):= \begin{cases}u_{t}\left(a, \Phi^{-1}(y)\right) & \text { if } y \in Y \\ 0 & \text { if } y \in \mathbb{R}^{m} \backslash Y\end{cases}
$$

For this game the internal-external form obtains by

$$
\begin{aligned}
u_{t}(a,(\mu \otimes \delta)(C \times \cdot) / \mu(C)) & =U_{t}(a, e(\delta)) \text { with } \\
e(\delta) & =\Phi((\mu \otimes \delta)(C \times \cdot) / \mu(C))=\left(I_{g_{a}}(\delta)\right)_{a \in A},
\end{aligned}
$$

where $g_{\bar{a}}(t, a):=1_{C}(t) 1_{\bar{a}}(a) / \mu(C)$. Observe here that $I_{g_{\bar{a}}}(\delta)=(\mu \otimes \delta)(C \times$ $\{\bar{a}\}) / \mu(C)$, similar to comments following Corollary 2.1 .

Theorem 3 in Khan et al. (2006) works with $C=T$ and is only stated for a mixed equilibrium profile $\delta$; then (i) and (ii) ensure that $f$ is a pure equilibrium profile in our setting by Remark 3.1(ii). Moreover, we observe that (Khan et al. 2006, Theorem 3) requires $u_{t}(a, \cdot)$ to be continuous on $\operatorname{Prob}(A)$ for every $t \in T$, so that even more can be said in that situation, because Remark 3.2 applies: so a pure equilibrium profile actually exists in this model.

\subsection{Non-atomic games in Mas-Colell's sense}

Also Mas-Colell's model of a non-atomic game Mas-Colell (1984), as studied in Sect. 6 of Khan et al. (2006) can be reformulated in internal-external form by making 
rather direct substitutions that were already explained in Balder (1991, 1996, 2002). Actually, we discuss a somewhat more general version of this model.

Let $A$ be a finite action set and let $T$ an arbitrary set of real-valued functions on $A \times \operatorname{Prob}(A)$ that forms a Borel-measurable subset of a complete separable metric space (think of such functions as the payoff functions, which are used here as players' types; thus they "mask" the players and give them a degree of anonymity). This is rather more general than Khan et al. (2006). Our condition for $T$ is certainly met if it is contained in the set $\mathcal{C}(A \times \operatorname{Prob}(A))$ of all continuous functions on $A \times \operatorname{Prob}(A)$ and if $T$ is measurable with respect to the Borel $\sigma$-algebra for the supremum norm on $\mathcal{C}(A \times \operatorname{Prob}(A))$; the model in (Khan et al. 2006, Sect. 6) is a further specialization of this situation (it has $T=\mathcal{C}(A \times \operatorname{Prob}(A)))$. Let $\mu$ be a non-atomic probability measure on $T$, the latter set being equipped with a $\sigma$-algebra $\mathcal{T}$ that has the following property: for every $a \in A$ and $v \in \operatorname{Prob}(A)$ the evaluation functional $u \mapsto u(a, v)$ on $T$ is $\mathcal{T}$-measurable (in particular, this is so in the above-mentioned special situation with $T \subset \mathcal{C}(A \times \operatorname{Prob}(A)))$. A $\mu$-compatible distribution over $T \times A$ is a probability measure $\tau$ on $T \times A$ such that $\left.\tau\right|_{T}=\mu$. An equilibrium distribution over $T \times A$ is a $\mu$-compatible distribution $\tau$ such that $\tau\left(B_{\tau}\right)=1$, where

$$
B_{\tau}:=\left\{(u, a) \in T \times A: a \in \operatorname{argmax}_{a^{\prime} \in A} u\left(a^{\prime},\left.\tau\right|_{A}\right)\right\}
$$

The following facts, which connect $\mu$-compatible distributions with mixed action profiles, were observed in Balder (1991, 1996, 2002):

(1) for every $\mu$-compatible [equilibrium] distribution $\tau$ there exists $\delta \in \mathcal{R}(T ; A)$, with $\tau=\mu \otimes \delta$, that is a mixed [equilibrium] action profile in the sense of Sect. 3 .

(2) if $\delta \in \mathcal{R}(T ; A)$ is a mixed [equilibrium] action profile in the sense of Sect. 3, then $\tau:=\mu \otimes \delta$ forms a $\mu$-compatible [equilibrium] distribution.

A $\mu$-compatible distribution $\tau$ is said to be symmetrizable if it is of the form $\tau=$ $\mu \otimes \epsilon_{f}$ for some $f \in \mathcal{M}(T ; A)$. With these substitutions and this terminology, application of Corollary 3.1 immediately gives the following:

Theorem 4.3 For every $\mu$-compatible distribution $\tau \in \operatorname{Prob}(T \times A), \tau=\mu \otimes \delta$, with $\delta \in \mathcal{R}(T ; A)$, there exists a symmetrizable $\mu$-compatible distribution $\mu \otimes \epsilon_{f}$, for $f \in \mathcal{M}(T ; A)$, with the following properties:

(i) $\left.\mu\left(f^{-1}(\cdot)\right)\right)=\tau(T \times \cdot)$,

(ii) $f(t) \in \operatorname{supp} \delta(t)$ for $\mu$-a.e. $t$ in $T$.

As a consequence of (i) and (ii), if $\tau$ happens to be an equilibrium distribution [this is a supposition in Theorem 4 of Khan et al. (2006)], then $\mu \otimes \epsilon_{f}$ is a symmetrizable equilibrium distribution-see Remark 3.1(ii).

To see that this result follows by Corollary 3.1, we let $Y$ and $\Phi$ be as in the previous subsection. We set $S:=A$ and $S_{t}:=A$ for all $t \in T$. Following an idea of Balder $(1991,1996)$ and (Balder 2002, Sect. 3.1) we set $U_{t}(a, y):=t\left(a, \Phi^{-1}(y)\right)$ for any $t \in T, a \in A, y \in Y$. Clearly, this gives the desired internal-external form

$$
U_{t}(a, e(\delta))=t\left(a,\left.\mu \otimes \delta\right|_{A}\right)
$$


if we set $e(\delta):=\Phi\left(\left.\mu \otimes \delta\right|_{A}\right)=(\mu(T \times\{a\}))_{a \in A}$, noting that $e(\delta)=\left(I_{g_{a}}(\delta)\right)_{a \in A}$. Here we use $g_{a}:(t, s) \mapsto 1_{\{a\}}(s)$; see Remark 3.2. Note that we work here with gph $\Sigma=T \times A$, so we have $\mathcal{R}_{\Sigma}=\mathcal{R}(T ; A)$ and $\mathcal{S}_{\Sigma}=\mathcal{M}(T ; A)$. Finally, we observe that the bonus mentioned in Remark 3.2 applies to the situation considered in Theorem 4 of Khan et al. (2006): this means that a symmetrizable equilibrium distribution actually exists there.

Acknowledgments The author is indebted to two anonymous referees for their very helpful and precise remarks.

\section{A Appendix}

In this appendix we recall a number of results that were cited in the main part of this paper. To begin with, recall that Lyapunov's classical theorem (Lyapunov 1940) on the range of a vector measure states the following:

Theorem A.1 (Lyapunov) For $k=1, \ldots, m$ let $\mu_{k}$ be a finite non-atomic measure on $(T, \mathcal{T})$. Then the range of the vector measure $\left(\mu_{k}\right)_{k=1}^{m}$, that is to say the set

$$
R:=\left\{\left(\mu_{1}(C), \ldots, \mu_{m}(C)\right): C \in \mathcal{T}\right\}
$$

is a convex, compact subset of $\mathbb{R}^{m}$.

Dvoretzky et al. (1950) gave the following result.

Theorem A.2 (Dvoretzky, Wald, Wolfowitz) For $k=1, \ldots, m$ let $\mu_{k}$ be a finite non-atomic measure on $(T, \mathcal{T})$. For $j=1, \ldots$, det $\delta_{j}: T \rightarrow \mathbb{R}_{+}$be such that $\sum_{j=1}^{d} \delta_{j}(t)=1$ for every $t \in T$. Then there exists a decomposition $\left\{C_{1}, \ldots, C_{d}\right\}$ of $T$ into $d$ disjoint measurable sets such that

$$
\int_{T} \delta_{j} d \mu_{k}=\mu_{k}\left(C_{j}\right) \text { for } j=1, \ldots, d \text { and } k=1, \ldots, m .
$$

This result extends Lyapunov's theorem: observe footnote 1 and note that for any $E, D \in \mathcal{T}$ and $\alpha \in(0,1)$ the six functions $\gamma 1_{D \backslash E}, \gamma 1_{D \cap E}, \gamma 1_{E \backslash D}$, with $\gamma=\alpha$ and $\gamma=1-\alpha$ form a measurable partition of unity, just as required in Theorem A.2. From an application of this result it easily follows that $\alpha\left(\mu_{k}(D)\right)_{k}+(1-\alpha)\left(\mu_{k}(E)\right)_{k}$ belongs to the range of the vector measure $\left(\mu_{k}\right)_{k}$, causing it to be a convex set. Theorem A.2 also extends the so-called bang-bang principle in optimal control theory (Hermes and Lasalle 1969, Theorem 8.2). This principle corresponds to the special case $d=2$ and because of (Hermes and Lasalle 1969, Theorem 8.1), this observation also shows how compactness as in Theorem A.1 is implied by Theorem A.2.

As already explained in the in the main text, Theorem A.2 follows directly from Theorem 2.1 as follows: (1) set $S:=\{1, \ldots, d\}$, (2) observe that the function $t \mapsto$ $\left(\delta_{1}(t), \ldots, \delta_{d}(t)\right)$ can be identified with a transition probability with respect to $(T, \mathcal{T})$ and $\left(S, 2^{S}\right)$ and (3) work with functions $g_{j}$ of the form $(t, s) \mapsto \frac{d \mu_{k}}{d \mu}(t) 1_{\bar{s}}(s)$. The 
resulting function $f$ of Theorem 2.1 then produces the desired decomposition of $T$ by setting $C_{j}:=f^{-1}(j)$.

The extension of Theorem A.2, as given by in (Khan et al. 2006, Corollary 1), is as follows:

Theorem A.3 For $k=1, \ldots, m$ let $\mu_{k}$ be a finite non-atomic probability measure on $(T, \mathcal{T})$. Let $S$ be a finite set. Let $g:=\left(g_{1}, \ldots, g_{d}\right): T \times S \rightarrow \mathbb{R}^{d}$ be $\mathcal{T} \otimes 2^{S}$ measurable and let $g_{j}(\cdot, s)$ be $\mu_{k}$-integrable for every $s \in S$ and $j=1, \ldots, d$. Also, let $\delta$ be a transition probability with respect to $(T, \mathcal{T})$ and $\left(S, 2^{S}\right)$. Then there exists a measurable function $f: T \rightarrow S$ such that for $k=1, \ldots, m$

(i) $\int_{T}\left[\int_{S} g_{j}(t, s) \delta(t)(d s)\right] \mu_{k}(d t)=\int_{T} g_{j}(t, f(t)) \mu_{k}(d t), j=1, \ldots, d$,

(ii) $\left(\mu_{k} \otimes \delta\right) \mid s=\mu_{k}\left(f^{-1}(\cdot)\right)$,

(iii) $f(t) \in \operatorname{supp} \delta(t)=\{s \in S: \delta(t)(\{s\})>0\}$.

As explained in the main text, this result follows directly from Theorem 2.1 (see Remark 2.1 and Corollary 2.1), in the same way as we showed above for Theorem A.2.

Aumann's identity for integrals of multifunctions Aumann (1965), which follows directly from Theorem A.2 by Carathéodory's theorem and measurable selection results (the latter largely due to Aumann himself), is as follows:

Theorem A.4 (Aumann) Let $\mu$ be a complete and finite non-atomic measure on $\left(T, \mathcal{T}\right.$. Let $F: T \rightarrow 2^{\mathbb{R}_{+}^{d}}$ be a multifunction whose graph is $\mathcal{T} \otimes \mathcal{B}\left(\mathbb{R}^{d}\right)$-measurable. Then

$$
\int_{T} F d \mu=\int_{T} F^{*} d \mu
$$

where $F^{*}(t)$ stands for the convex hull of the set $F(t)$ and where for $H=F, F^{*}$

$$
\int_{T} H d \mu:=\left\{\int_{T} h d \mu: h \in \mathcal{H}\right\},
$$

with $\mathcal{H}$ the set of all $\mu$-integrable functions $h: T \rightarrow \mathbb{R}_{+}^{d}$ with $h(t) \in H(t)$ for all $t \in T$.

The original result in Aumann (1965) was stated for the unit interval T cum Lebesgue structure, but its proof easily extends to the form given here. Moreover, it is well-known (Castaing and Valadier 1977) that in the above result the completeness condition for the measure $\mu$ can be dropped by redefining $\mathcal{H}$ to be the set of all $\mu$-integrable $h$ for which $h(t) \in H(t)$ holds for almost every $t \in T$.

Suppose now that in Sect. 3, in addition to the assumptions already in place there, the following hold for every $t \in T$ :

(i) the set $S_{t}$ is compact,

(ii) the function $U_{t}(\cdot, \cdot)$ is upper semicontinuous on $S_{t} \times \mathbb{R}^{m}$,

(iii) the function $y \mapsto \sup _{s \in S_{t}} U_{t}(s, y)$ is lower semicontinuous on $\mathbb{R}^{m}$. 
Suppose also in addition that the measure space $(T, \mathcal{T}, \mu)$ is separable. Then the pure equilibrium existence result of (Balder 2002, Theorem 2.2.1), referred to in Remark 3.2 above, is as follows when it is specialized to the non-atomic case of this paper (see the observations made at the beginning of Sect. 3).

Theorem A.5 There exists $f \in \mathcal{S}_{\Sigma}$ that is a pure Cournot-Nash equilibrium profile in the sense of (4).

\section{References}

Angeloni L, Martins-da-Rocha F (2005) A comment on Nash equilibrium existence results for large games with summary statistics. Working paper

Ash RB (1972) Real analysis and probability. Academic, New York

Aumann RJ (1965) Integrals of set-valued functions. J Math Anal Appl 12:1-12

Balder EJ (1984) A general denseness result for relaxed control theory. Bull Aust Math Soc 30:463-475

Balder EJ (1985) Elimination of randomization in statistical decision theory reconsidered. J Multivariate Anal 16:260-264

Balder EJ (1988) Generalized equilibrium results for games with incomplete information. Math Oper Res 13:265-276

Balder EJ (1991) On Cournot-Nash equilibrium distributions for games with differential information and discontinuous payoffs. Econ Theory 1:339-354

Balder EJ (1995) A unifying approach to existence of Nash equilibria. Int J Game Theory 24:79-94

Balder EJ (1996) Comments on the existence of equilibrium distributions. J Math Econ 25:307-323

Balder EJ (1999) On the existence of Cournot-Nash equilibria in continuum games. J Math Econ 31:207223

Balder EJ (2000) Lectures on Young measure theory and its applications in economics. Rend Istit Mat Univ Trieste 31 Suppl. 1:1-69. Electronically available at http://www.dmi.units.it/ rimut/volumi/ 31_supp1/index.html

Balder EJ (2000) Incompatibility of usual conditions for equilibrium existence in continuum economies without ordered preferences. J Econ Theory 93:110-117

Balder EJ (2002) A unifying pair of Cournot-Nash equilibrium existence results. J Econ Theory 102:437470

Balder EJ (2004) On infinite-dimensional generalizations of Lyapunov's convexity theorem and Aumann's identity. Working paper

Berliocchi H, Lasry JM (1973) Intégrandes normales et mesures paramétrées en calcul des variations. Bull Soc Math France 101:129-184

Castaing C, Valadier M (1977) Convex analysis and measurable multifunctions. Springer, Berlin

Dellacherie C, Meyer, PA (1978) Probabilities and potential. North-Holland Amsterdam

Dvoretzky A, Wald A, Wolfowitz J (1950) Elimination of randomization in certain problems of statistics and the theory of games. Proc Nat Acad Sci USA 36:256-260

Hermes H, Lasalle JP (1969) Functional analysis and time optimal control. Wiley, New York

Khan MA, Rath KP, Sun Y (2006) The Dvoretzky-Wald-Wolfowitz theorem and purification in atomless finite-action games. Int J Game Theory 34:91-104

Khan MA, Sun Y (1996) Integrals of set-valued functions with a countable range. Math Oper Res 21:946-954

Kingman JFC, Robertson AP (1968) On a theorem of Lyapunov. J Lond Math Soc 43:347-351

Lyapunov A (1940) Sur les fonctions-vecteurs complètement additives. Izvestiya Akad Nauk SSSR Ser Mat 4:465-478

Loeb P, Sun Y (2006) Purification of measure-valued maps. Ill J Math 50:747-762

Martins-da-Rocha F, Topuzu M (2006) Cournot-Nash equilibria in continuum games with non-ordered preferences (submitted)

Mas-Colell A (1984) On a theorem of Schmeidler. J Math Econ 13:201-206

Milgrom PR, Weber RJ (1985) Distributional strategies for games with incomplete information. Math Oper Res 10:619-632

Neveu J (1965) Mathematical foundations of the calculus of probability. Holden-Day, San Francisco 
Podczeck K (2003) Core and Walrasian equilibria when agents' characteristics are extremely dispersed. Econ Theory 22:699-725

Podczek K (2006) A note on purification of measure-valued maps. Working paper

Radner R, Rosenthal RW (1982) Private information and pure-strategy equilibria. Math Oper Res 7:401-409

Rustichini A, Yannelis NC (1991) What is perfect competition? In: Khan MA, Yannelis NC (eds.) Equilibrium theory in infinite dimensional spaces. Springer, Berlin, pp 249-265

Schmeidler D (1973) Equilibrium points of non-atomic games. J Stat Phys 7:95-300

Shafer W, Sonnenschein H (1975) Equilibrium in abstract economies without ordered preferences. J Math Econ 2:345-348

Tourky R, Yannelis NC (2001) Markets with many more agents than commodities: Aumann's "hidden" assumptions. J Econ Theory 101:189-221 